Document downloaded from:

http://hdl.handle.net/10251/154811

This paper must be cited as:

Gimenez, JJ.; Carcel, JL.; Fuentes, M.; Garro, E.; Elliott, S.; Vargas, D.; Menzel, C.... (2019). 5G New Radio for Terrestrial Broadcast: A Forward-Looking Approach for NRMBMS. IEEE Transactions on Broadcasting. 65(2):356-368.

https://doi.org/10.1109/TBC.2019.2912117

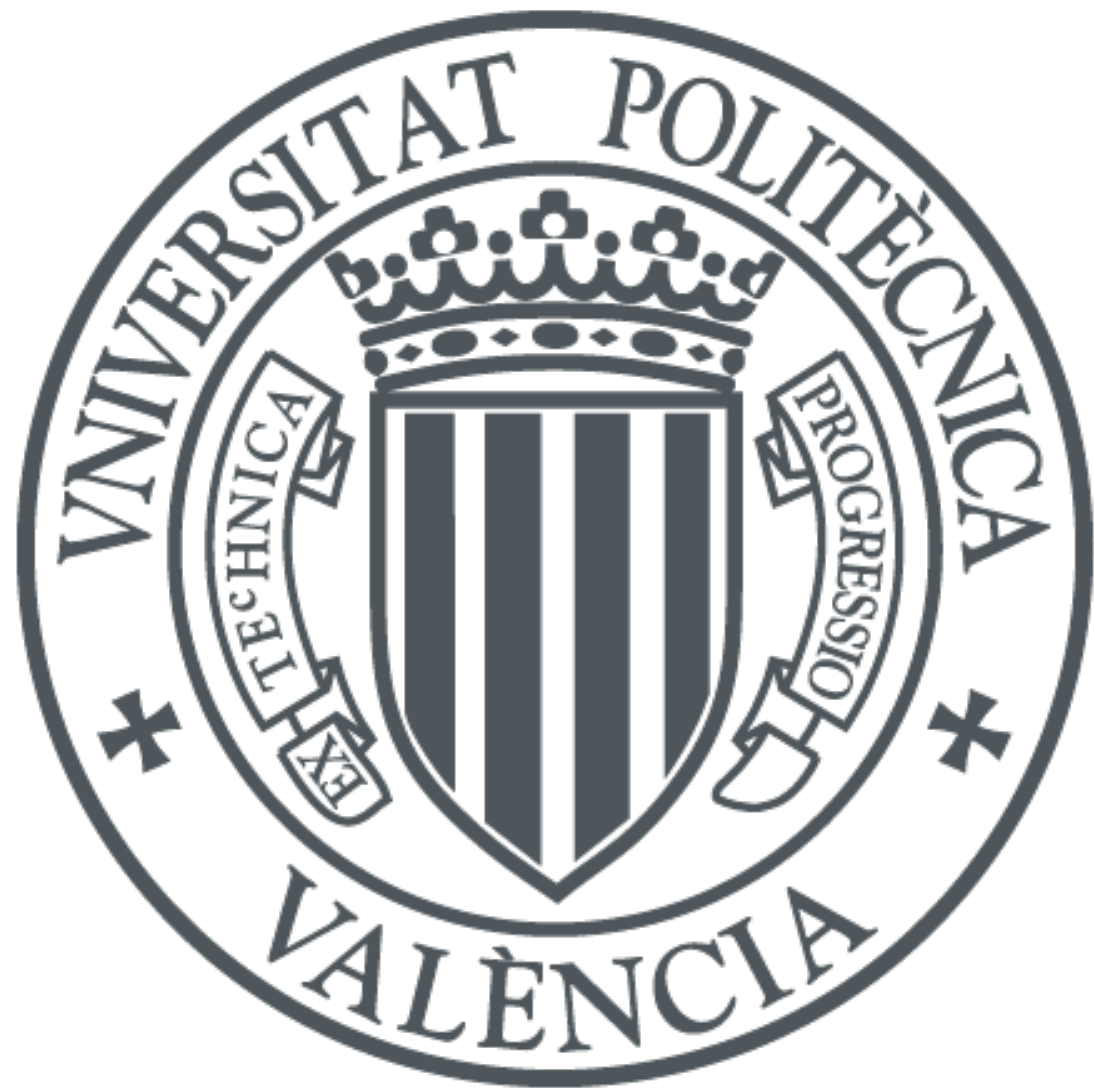

The final publication is available at

https://doi.org/10.1109/TBC.2019.2912117

Copyright Institute of Electrical and Electronics Engineers

Additional Information

"(C) 2019 IEEE. Personal use of this material is permitted. Permissíon from IEEE must be obtained for all other uses, in any current or future media, including reprinting/republishing this material for advertisíng or promotional purposes, creating new collective works, for resale or redistribution to servers or lists, or reuse of any copyrighted component of this work in other works." 


\title{
5G New Radio for Terrestrial Broadcast: A Forward-Looking Approach for NR-MBMS
}

\author{
Jordi Joan Gimenez, Jose Luis Carcel, Manuel Fuentes, Eduardo Garro, Simon Elliott, David Vargas, \\ Christian Menzel and David Gomez-Barquero
}

\begin{abstract}
GPP LTE eMBMS Release 14, also referred to as FeMBMS (Further evolved Multimedia Broadcast Multicast Service) or EnTV (Enhanced TV), is the first mobile broadband technology standard to incorporate a transmission mode designed to deliver Terrestrial Broadcast services from conventional High Power High Tower (HPHT) broadcast infrastructure. With respect to the physical layer, the main improvements in FeMBMS are the support of larger inter-site distance for Single Frequency Networks (SFN) and the ability to allocate $100 \%$ of a carrier's resources to the broadcast payload, with self-contained signaling in the downlink. From the system architecture perspective, a receive-only mode enables free-to-air (FTA) reception with no need for an uplink or SIM card, thus receiving content without $\mathrm{UE}$ registration with a network. These functionalities are only available in the LTE Advanced Pro specifications as 5G New Radio (NR), standardized in 3GPP from Release 15, has so far focused entirely on unicast. This paper outlines a physical layer design for NR-MBMS, a system derived, with minor modifications, from the 5G-NR specifications, and suitable for the transmission of linear TV and radio services in either single-cell or SFN operation. The paper evaluates the NRMBMS proposition and compares it to LTE-based FeMBMS in terms of flexibility, performance, capacity and coverage.
\end{abstract}

Index Terms-MBMS, eMBMS, FeMBMS, DTT, MBSFN, SCPTM, LTE, 5G, New Radio, NR

\section{INTRODUCTION}

$\mathrm{T}$ errestrial Broadcast, as a 3GPP use case, was first addressed in LTE Advanced Pro 3GPP Release (Rel-) 14

This work was partially supported by the European Commission under the 5G-PPP project 5G-Xcast (H2020-ICT-2016-2 call, grant number 761498). The views expressed in this contribution are those of the authors and do not necessarily represent those expressed in the 5G-Xcast project or the author's organizations.

Parts of this paper have been published in the Proceedings of the IEEE BMSB 2018, Valencia, Spain.

J.J. Gimenez and C. Menzel are with the Institut für Rundfunktechnik GmbH, Floriansmuhlstrasse 60, 80939 Munich, Germany (e-mail: \{jordi.gimenez, christian.menzel\}@ irt.de).

J.L. Carcel is with Samsung Electronics R\&D UK, Communication House South Street, Staines-Upon-Thames TW18 4QE, United Kingdom (e-mail: jose.cervera@ samsung.com).

M. Fuentes, E. Garro and D. Gomez-Barquero are with the Univesitat Politècnica de València, Camí de Vera SN, 46022, Valencia, Spain (e-mail: \{mafuemue, edgarcre, dagobar\} @iteam.upv.es).

S. Elliott is with BBC Distribution and Business Development, White City Place, 201 Wood Lane London, W12 7TQ, England, (e-mail: simon.elliott@bbc.co.uk).

D. Vargas is with BBC Research and Development, The Lighthouse, BBC Broadcast Centre, 16 Alliance Court, Alliance Road, Acton, London W3 0RB, England, (e-mail: david.vargas@bbc.co.uk). in which the Multimedia Broadcast Multicast Service (MBMS) system was enhanced to operate in a dedicated mode for the delivery of linear broadcast services (i.e.radio and TV), fulfilling a wide set of requirements input by the broadcast industry [1]. 3GPP's Enhancements for TV (EnTV) study item proposed several enhancements resulting in the FeMBMS (further evolved MBMS) of Rel-14. In order to leverage the well-established and proven LTE ecosystem, it was decided to base FeMBMS on the pre-existing LTE Advanced Pro specifications with enhancements being made as necessary in order to fulfill the requirements. Enhancements made to the system architecture comprise: (i) the XMB interface through which broadcasters can establish the control and data information of audio-visual services; (ii) a new Application Programming Interface (API) for developers to simplify access to eMBMS procedures in the User Equipment (UE); (iii) the support of multiple media codecs and formats; (iv) a transparent delivery mode to support native content formats over IP without transcoding (e.g. reusing existing MPEG-2 Transport Streams and compatible equipment); (v) the support of shared eMBMS broadcast by aggregating different eMBMS networks into a common distribution platform; and (vi) the receive-only mode (ROM), which enables devices to receive broadcast content with no need for uplink capabilities, SIM cards or network subscriptions - i.e. free-to-air reception.

From the radio layer point of view the most significant enhancements are: (i) the possibility to establish dedicated FeMBMS carriers that allocate up to $100 \%$ of the radio resources to Terrestrial Broadcast (i.e. with no frequency or time multiplexing with unicast resources in the same frame), self-contained signaling and system information in the downlink; (ii) a new, reduced overhead subframe containing no unicast control region; and (iii) the support of larger inter-site distances in SFN (Single Frequency Networks) reaching higher spectral efficiency with a new numerology $-1.25 \mathrm{kHz}$ subcarrier spacing (SCS) and $200 \mu$ s cyclic prefix (CP). The new numerology changes are the most significant as the longer OFDM symbol duration, occupying one subframe, made it necessary to design a new subframe structure, known as the CAS (Cell Acquisition Subframe), to allocate the synchronization and control channels, transmitted with much reduced periodicity (one in every forty subframes) [2].

These changes led to a system like other Digital Terrestrial Broadcast systems such as DVB-T/T2 [3], ATSC 3.0 [4] or $\mathrm{DAB} / \mathrm{DAB}+$ [5]. In addition to broadcast content, mobile broadband subscribers who have a SIM card can enjoy enriched service offerings when combined with independent unicast for interactivity, in a similar way to conventional 
TABLE I. NUMEROLOGY OPTIONS IN FEMBMS

\begin{tabular}{ccccccc}
\hline \hline Type & $\begin{array}{c}\boldsymbol{\Delta}_{\boldsymbol{f}} \\
(\mathrm{kHz})\end{array}$ & $\mathrm{SC}_{\mathrm{RB}}$ & $\begin{array}{c}\text { OFDM } \\
\text { symbols } \\
\text { per SF }\end{array}$ & $\begin{array}{c}T_{C P} \\
(\mu \mathrm{s})\end{array}$ & $T_{U}(\mu \mathrm{s})$ & $\begin{array}{c}\text { ISD } \\
(\mathrm{km})\end{array}$ \\
\hline SC-PTM & 15 & 12 & 14 & $4.7 / 5.1$ & 66.7 & 1.4 \\
& 15 & 12 & 12 & 16.7 & 66.7 & 5 \\
MBSFN & 7.5 & 24 & 6 & 33.3 & 133.3 & 10 \\
& 1.25 & 144 & 1 & 200 & 800 & 60 \\
\hline \hline
\end{tabular}

Note that a Resource Block (RB) in LTE is $180 \mathrm{kHz}$ wide in frequency and 1 slot long in time. $\mathrm{SCRB}=$ Subcarriers per Resource Block, $\mathrm{SF}=$ Subframe, $\mathrm{T}_{\mathrm{CP}}=\mathrm{CP}$ duration, $\mathrm{T}_{\mathrm{U}}=$ useful OFDM symbol duration, ISD =SFN Inter-Site Distance.

HbbTV (Hybrid Broadcast Broadband TV) sets [6]. The introduction of a ROM and the new framing and numerology options may make FeMBMS suitable for use with conventional broadcast infrastructure (including high, medium and low power sites).

A further study item in 3GPP Rel-16 [7] has evaluated the ability of FeMBMS to support SFN of cells with coverage radii of up to $100 \mathrm{~km}$ (implying even longer CP) and mobile reception with speeds up to $250 \mathrm{~km} / \mathrm{h}$ (large SCS). A wider range of numerologies, supporting multiple network topologies, capacity improvements from longer symbol durations (which reduce CP overheads), new reference signals (RS) and greater bandwidth occupancy were also in the scope of the study. The benefits of time interleaving [8] and LDM (Layered Division Multiplexing) [9], also known as MUST (Multiuser Superposition Transmission) [10], were also taken into consideration. The signal acquisition and synchronization procedures were also evaluated as the existing numerology mismatch between data and control channels for large SFNs may lead to coverage issues as reported in [11] and [12]. Based on the findings of the Study Item, a Work Item may then standardize further improvements in Rel-16 while taking into account practical considerations such as implementation complexity and performance.

In parallel, 3GPP is standardizing 5G New Radio (NR) and 5G Core (5GC) specifications, with a new and more efficient radio layer and flexible system architecture. However, NR Rel15 and Rel-16 have so far focused on unicast. After the RAN\#79 plenary meeting, the broadcast work was split into two tracks, one to design a mixed unicast/multicast/broadcast mode for NR and another for LTE-based dedicated Terrestrial Broadcast. Although the latter has become known as LTEbased 5G Terrestrial Broadcast, the system is not based on 5GNR.

Several attempts have been made to start a work item for NR-MBMS, but these were not sufficiently supported to take them any further. [13], for example, proposed the introduction of extended CPs based on NR numerology, without success.

Nevertheless, work on NR looks set to continue into 2019, bringing an opportunity to introduce new functionalities for Terrestrial Broadcast operation with enhanced flexibility and scalability over LTE-based specifications which are subject to legacy constraints.

This paper outlines a physical layer design for a new NRMBMS system based on an extension of NR Rel-15. The design has little impact on the existing unicast mode, achieved by considering Terrestrial Broadcast services as a configuration option in which, simply, up to $100 \%$ of the $5 \mathrm{G}$ mixed mode carrier resources may be allocated to linear TV/radio services. The design is based on flexible numerology, framing and bandwidth configurations in order to cater many different types of networks ranging from single cell deployments to nationwide SFN (the latter requiring a more complex design), and reception environments from fixed rooftop to mobile. The proposed design has been evaluated in terms of Doppler performance, coverage, SFN echo tolerance and acquisition of system information and synchronization. As broadcast is not yet part of 5G-NR, several assumptions have been made, considering that other parts of the NR-MBMS system may enable the expected functionalities.

The rest of the paper is organized as follows: Section II provides a brief introduction of the physical layer characteristics of FeMBMS that are relevant for Terrestrial Broadcast. Section III then introduces the details of 5G-NR Rel-15 that have been further developed to support Terrestrial Broadcast in NR-MBMS. Section IV evaluates and compares FeMBMS and NR-MBMS solutions. Section V concludes the paper.

\section{BACKGROUND: FEMBMS PHYSICAL LAYER DESIGN FOR 5G TERRESTRIAL BROADCAST}

Since its introduction in LTE Rel-9, eMBMS has generally been associated with SFN operation in LPLT cellular networks with the Multicast Broadcast SFN (MBSFN) mode. From Rel13, eMBMS added another type of radio bearer, known as SCPTM (single-cell point-to-multipoint), that does not offer SFN capability but uses the unicast physical data channel with the regular $\mathrm{CP}$ to withstand echo delays due to multipath. Both types of bearers can be configured for Terrestrial Broadcast in FeMBMS (i.e. the allocation of up to $100 \%$ of the radio resources) enabling their respective functionalities. Their main physical layer characteristics are explained below.

\section{A. Numerology options for LTE FeMBMS}

Different numerology options are available for FeMBMS operation.

Within an MBSFN area, all the constituent transmitters must deliver the same content at the same time, on the same frequency. The CP appended to the beginning of the OFDM symbol avoids Inter-Symbol Interference (ISI), provided that all signals are received with relative delays shorter than the $\mathrm{CP}$ duration. As set out in Table I, FeMBMS has three values of extended $\mathrm{CP}\left(T_{C P}\right): 16.66 \mu \mathrm{s}, 33.33 \mu \mathrm{s}$ and $200 \mu \mathrm{s}$. The latter requires a $1 \mathrm{~ms}$ OFDM symbol duration - an entire subframe ${ }^{1}$. The SCS $\left(\Delta_{f}=1 / T_{U}\right)$, and corresponding OFDM symbol duration $\left(T_{U}\right)$ for these three CPs are also shown in Table I. Importantly, the new, much longer $200 \mu \mathrm{s}$ CP of Rel-14 has extended the maximum inter-site distance (ISD) in a network to around $60 \mathrm{~km}$, thus potentially supporting MBSFN areas in HPHT networks.

In all cases the overhead due to the $\mathrm{CP}$ is $20 \%$. The reason for the comparatively large overhead comes from a design based on mobile reception, where a compromise is required between capacity (that would increase by reducing the ratio

\footnotetext{
${ }^{1}$ In LTE, a $10 \mathrm{~ms}$ frame is divided into 10 subframes, each of $1 \mathrm{~ms}$ duration. In turn, each subframe is divided into two $0.5 \mathrm{~ms}$ slots. Each slot comprises one Resource Block (RB) in time. In frequency, each RB occupies $180 \mathrm{kHz}$, equivalent to 12 consecutive OFDM subcarriers for the normal $\mathrm{CP}$ configuration (15 KHz SCS).
} 
$T_{C P} / T_{U}$ ) and resilience to Doppler spread (greater robustness with higher $\Delta_{f}$ ).

SC-PTM does not support SFN, and may only be configured with the conventional 'unicast' CPs designed to cope with multipath. The overhead for the normal $\mathrm{CP}$ is around $7 \%$. Another difference between SC-PTM and MBSFN is that SC-PTM data is scheduled using the same physical data channel as for unicast traffic (PDSCH - Physical Downlink Shared Channel) while MBSFN data is scheduled using an ad-hoc physical channel that enables the extended CP (PMCH - Physical Multicast Channel).

\section{B. Reference Signals}

The MBSFN modes have a set of reference signal (MBSFN RS) patterns that are denser in the frequency domain compared to the standard unicast patterns in order to help receivers cope with the higher frequency selectivity caused by echoes received from distant transmitters in SFNs. Each base-station (i.e. cell) belonging to an MBSFN area transmits the same MBSFN reference signal pattern at the same time-frequency position in the $\mathrm{PMCH}$.

As shown in Fig. 1, for the MBSFN $15 \mathrm{kHz}$ SCS, reference symbols are inserted in every other sub-carrier in OFDM symbols 3, 7 and 11 of each sub-frame, with a single subcarrier offset in the OFDM symbol number 7. For $7.5 \mathrm{kHz}$ SCS, one reference signal is inserted in every four sub-carriers in OFDM symbol numbers 2, 4 and 6 of each sub-frame, with a two subcarrier offset in OFDM symbol 4. In the $1.25 \mathrm{kHz}$ variant, one reference signal is allocated every six subcarriers, with an offset of 3 subcarriers between odd and even sub-frames.

With respect to multipath or echoes - either artificial or natural - the frequency spacing between reference signals determines the length of delay up to which the channel may be correctly equalized when using time-frequency interpolation. Delays up to the duration of the equalization interval (EI) may be tolerated.

The EI is calculated assuming that the receiver is able to perform time and frequency interpolation. A factor of 57/64 is considered to account for realistic receiver implementation [14]. According to the frequency separation between reference signals $D_{f}$, the EI $\left(57 / 64 * T_{U} / D_{f}\right)$ for MBSFN subframes is 59.3 $\mu \mathrm{s}$ for $\Delta_{f}=\{15,7.5\} \mathrm{kHz}$ and $237.5 \mu \mathrm{s}$ for $\Delta_{f}=1.25 \mathrm{kHz}$. The overheads due to RS are $12.5 \%(15$ and $7.5 \mathrm{kHz})$ and $16.6 \%(1.25 \mathrm{kHz})$. Note that in the figure, $D_{t}$ is the length of the RS pattern in OFDM symbols.

For SC-PTM, cell-specific reference signals (C-RS) are used, for channel estimation of the PDSCH, with the patterns shown in Fig.1 It can be seen that they are sparser, and therefore overheads reduce at $4.7 \%(15 \mathrm{kHz}$ normal $\mathrm{CP})$ and $5.5 \%(15 \mathrm{kHz}$ extended $\mathrm{CP})$.

\section{Control Channels - Cell Acquisition Subframe (CAS)}

Previous releases of LTE eMBMS defined MBSFN frames with up to $60 \%$ broadcast resource allocation (up to 6 out of 10 subframes in each frame could be allocated to broadcast as two were permanently designated for paging and two more for synchronization). The capacity not allocated to broadcast could be allocated to unicast traffic. The latest modifications in FeMBMS enabled the configuration of up to $80 \%$ broadcast resource allocation and also a dedicated carrier with almost
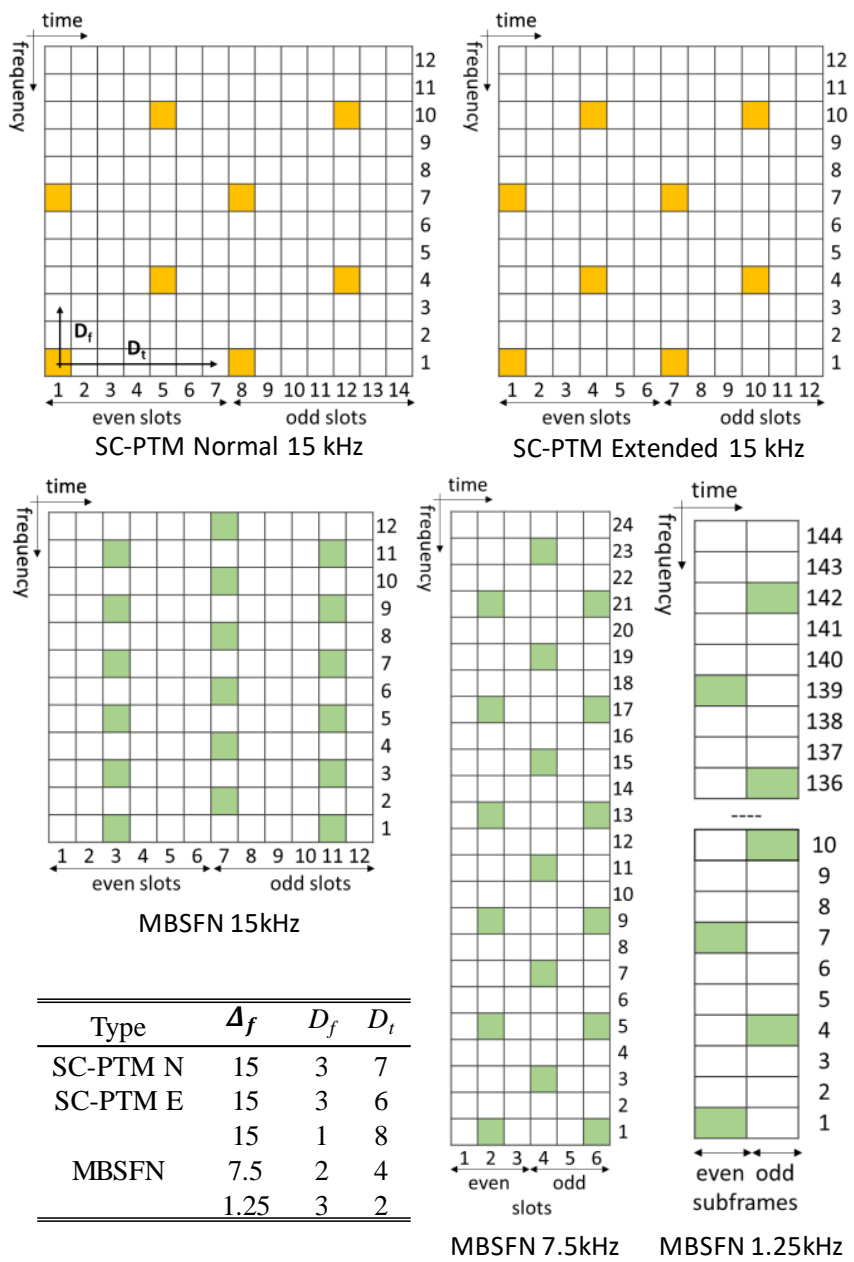

Fig. 1. Reference signals for MBSFN subframes and unicast subframes with different numerologies.

$100 \%$ broadcast allocation $(97.5 \%)$, by minimizing the signaling required for synchronization, acquisition and system information and moving it into the newly defined Cell Acquisition Subframe (CAS) that is transmitted once every 40 subframes (i.e. $2.5 \%$ signaling overhead). SC-PTM carriers are more flexible as they can multiplex broadcast data with higher time and frequency granularity - as FDM is possible, there is no need to dedicate complete subframes to broadcast.

The CAS is formed of the following physical signals and channels:

- PSS (Primary Synchronization Signal): symbol timing and partial physical cell identity (PCI) information

- SSS (Secondary Synchronization Signal): frame timing, transmission mode, $\mathrm{CP}$ duration and complete PCI.

- CS-RS (Cell-Specific Reference Signal): amplitude and phase reference for channel estimation.

- PBCH (Physical Broadcast Channel): Master Information Block (MIB), number of TX antennas, downlink BW and system frame number.

- PCFICH (Physical Control Format Indicator Channel): number of OFDM symbols used for control for each subframe.

- PDCCH (Physical Downlink Control Channel): downlink control indicator (DCI), transmission parameters and scheduling. 
- PDSCH (Physical Downlink Shared Channel): System Information Blocks (SIBs).

The CAS may only be configured with numerologies defined for unicast (i.e. $15 \mathrm{kHz}$ SCS with or without extended CP). From the point of view of Terrestrial Broadcast, the CAS acts as a preamble containing physical layer signaling (similar to P1 and P2 symbols in DVB-T2 or bootstrap and preambles in ATSC 3.0). It is important to note that each service transmitted in MBMS is identified with a corresponding Temporary Mobile Group Identifier (TMGI) ${ }^{2}$. With the information obtained from the CAS and the TMGI, the UE can disclose an on-going MBMS session and receive the service. When SC-PTM is configured, data is scheduled over the PDSCH according to a specific Radio Network Temporary Identifier (RNTI), reusing the unicast mechanism to schedule user data. For broadcast a Group RNTI (G-RNTI) is used, which is then mapped to a TMGI.

\section{Identified limitations of FeMBMS}

Although the LTE FeMBMS improvements standardised in Rel-14 allow the delivery of linear broadcast services (such as TV and radio) with a dedicated broadcast mode, the specifications lack flexibility in parameters such as the $\mathrm{CP}, T_{U}$ duration, and FFT size to adapt the system to the wide range of broadcasting network topologies deployed around the world:

- For fixed-rooftop reception, HPHT SFN networks with very large ISD (circa $150 \mathrm{~km}$ ) may benefit from CPs greater than $200 \mu \mathrm{s}$.

- For mobile environments with high speed requirements (e.g. $250 \mathrm{~km} / \mathrm{h}$ ) in sparse LPLT networks, such as in rural areas, numerologies with a CP between 33 and $200 \mu \mathrm{s}$ options may be beneficial.

Large inter-site distances and high mobility with speeds up to $250 \mathrm{~km} / \mathrm{h}$ are requirements set out in $3 \mathrm{GPP}$ [1] for the $5 \mathrm{G}$ physical layer to deliver broadcast and multicast services.

Another limitation is the fixed CP overhead of $20 \%$ which cannot be modified to suit static or mobile scenarios. In fixed rooftop environments where the Doppler spread does not impose a significant limitation, narrower SCSs could be used. These would increase $T_{U}$ in order to lower the $\mathrm{CP}$ overhead for a fixed absolute CP length. Even more, FeMBMS, and in particular SC-PTM, has not been optimized for single-cell or MFN networks, where overheads could be reduced.

Another important aspect is the performance of the CAS subframe due to the aforementioned numerology mismatch (i.e. the CAS can only use CP lengths that are shorter than the data subframes). Thus, CAS subframes may limit the final system performance in networks where significant echoes are received with delays greater than $16 \mu \mathrm{s}$.

Furthermore, multiple input multiple output (MIMO) techniques with spatial multiplexing are not supported in FeMBMS [2], which limits the potential maximum capacity of the system.

Potential inefficiencies are also identified. Service information in MBMS is carried over multiple control channels. SIBs are not only transmitted in the PDSCH of the CAS but also in the logical MCCH (Multicast Control

\footnotetext{
${ }^{2}$ Note that for ROM receivers, a range of TMGIs with MCC $=901$ and $\mathrm{MNC}=56$ is standardized so that there is no need to register to an operator.
}

Channel) and SC-MCCH channels. These are multiplexed with service data in the MTCH (Multicast Traffic Channel) or SC$\mathrm{MTCH}$, and transmitted in the $\mathrm{PMCH}$ or the $\mathrm{PDSCH}$, respectively for MBSFN or SC-PTM. In addition to a distinct downlink channel in MBSFN, service information depends on the type of carrier and deployment.

\section{A NEW PHysical LAYER DESIGN FOR 5G TERRESTRIAL BROADCAST BASED ON NEW RADIO (NR-MBMS)}

5G-NR offers a more flexible and scalable design than LTE in order to support a wider range of use cases requirements, including an extensive range of frequency bands and deployment options. However, NR Rel-15 and Rel-16 have considered only unicast. Transmission modes and core functionality do not yet support broadcast or multicast. Opportunity therefore exists in NR to define transmission modes suitable for Terrestrial Broadcast with fewer constraints compared with FeMBMS, which is based on well-established LTE specifications.

This section explains the main characteristics of 5G-NR already specified for unicast and explores their potential extension for Terrestrial Broadcast.

\section{A. 5G-NR physical layer design}

5G-NR is based on a CP-OFDM solution similar to LTE. The main new feature is that the $5 \mathrm{G}$ waveform is combined with a scalable numerology that enables radio resource allocation over different frequency bands. The SCS is scaled according to $15 \times 2^{\mu} \mathrm{kHz}$, where $15 \mathrm{kHz}$ is taken as the base SCS (as for LTE) and $2^{\mu}$ generates additional numerology options. Five different numerologies are defined with SCS from $15 \mathrm{kHz}$ to $240 \mathrm{kHz}$ (from $\mu=0$ to 4 ). Note that the parameter $\mu$ is only defined for positive integer numbers. The possible values vary with the frequency band in order to cope with Doppler spread (higher at higher frequencies), and to extend the bandwidth, greater at high frequencies. This scalable method permits the different numerology options to be aligned in the time domain as extensions of basic slots and OFDM symbols.

The most significant change with respect to LTE is that one slot always contains 14 OFDM symbols for all different SCS values. Therefore, the number of slots per subframe (and frame) increases for wider SCS and an RB is defined as 12 subcarriers in only one OFDM symbol in the time domain (rather than one slot as per LTE). With respect to the system bandwidth, 5G-NR brings the possibility to configure larger bandwidth carriers (e.g., $100 \mathrm{MHz}$ or $400 \mathrm{MHz}$ ) than LTE. Note that different numerologies can be multiplexed within the same NR carrier bandwidth both using Time (TDM) and Frequency Division Multiplexing (FDM) thanks to a new concept called Carrier Bandwidth Part (CBP). Using CBP it is possible to define groups of consecutive RBs, including different numerologies, over the same carrier. A maximum of 4 BW parts can be specified, which may be enough for the purpose of multiplexing different numerologies from a single wideband transmitter.

5G NR also introduces some variations with respect to control channels. In particular, the frame structure avoids the mapping of control channels across the full carrier bandwidth, 
TABLE II. NUMEROLOGY OPTIONS FOR 5G TERRESTRIAL BROADCAST

\begin{tabular}{ccccccccc}
\hline \hline & $\mu$ & $\boldsymbol{\Delta}_{\boldsymbol{f}}(\mathrm{Hz})$ & $T_{U}(\mu \mathrm{s})$ & $\begin{array}{c}\mathrm{CP} \\
\text { Fraction }\end{array}$ & $\begin{array}{c}T_{C P} \\
(\mu \mathrm{s})\end{array}$ & $\begin{array}{c}T_{S} \\
(\mathrm{~ms})\end{array}$ & $\mathrm{SC}_{\mathrm{RB}}$ & $\begin{array}{c}\text { ISD } \\
(\mathrm{km})\end{array}$ \\
\hline $\mathrm{A}$ & 0 & 15000 & 66.67 & $\sim 7 \%$ & $4.7 / 5.1$ & 0.07 & 12 & 1.4 \\
$\mathbf{B}$ & $\mathbf{0}$ & $\mathbf{1 5 0 0 0}$ & 66.67 & $20 \%$ & 16.67 & 0.08 & 12 & 5 \\
$\mathbf{C}$ & $\mathbf{- 1}$ & $\mathbf{7 5 0 0}$ & 133.33 & $20 \%$ & 33.33 & 0.17 & 24 & 10 \\
$\mathbf{D}$ & $\mathbf{- 2}$ & $\mathbf{3 7 5 0}$ & 266.67 & $20 \%$ & 66.67 & 0.33 & 48 & 20 \\
$\mathrm{E}$ & - & 2500 & 400.00 & $20 \%$ & 100.00 & 0.50 & 72 & 30 \\
$\mathbf{F}$ & $\mathbf{- 3}$ & $\mathbf{1 8 7 5}$ & 533.33 & $20 \%$ & 133.33 & 0.67 & 96 & 40 \\
$\mathrm{G}$ & - & 1250 & 800.00 & $20 \%$ & 200.00 & 1.0 & 144 & 60 \\
$\mathrm{H}$ & - & 625 & 1600.00 & $20 \%$ & 400.00 & 2.0 & 288 & 120 \\
$\mathrm{I}$ & - & 3333 & 300.00 & $10 \%$ & 33.33 & 0.33 & 54 & 10 \\
$\mathrm{~J}$ & - & 2045.45 & 488.88 & $2.22 \%$ & 11.11 & 0.50 & 88 & 3.3 \\
$\mathrm{~K}$ & - & 1022.72 & 977.78 & $2.22 \%$ & 22.22 & 1.0 & 176 & 6.6 \\
$\mathrm{~L}$ & - & 511.36 & 1955.56 & $2.22 \%$ & 44.44 & 2.0 & 352 & 13.2 \\
$\mathrm{M}$ & - & 416.67 & 2400 & $4 \%$ & 100 & 2.5 & 432 & 30 \\
$\mathrm{~N}$ & - & 208.33 & 4800 & $4 \%$ & 200 & 5.0 & 864 & 60 \\
$\mathrm{O}$ & - & 104.67 & 9600 & $4 \%$ & 400 & 10.0 & 1728 & 120 \\
$\mathrm{p}$ & - & 217.39 & 4600 & $8 \%$ & 400 & 5.0 & 828 & 120 \\
\hline \hline
\end{tabular}

$\mathrm{SC}_{\mathrm{RB}}=$ Subcarriers per Resource Block

giving more flexibility than LTE and enabling the selection of the position of control channels across the frequency domain. In this case, PSS, SSS and PBCH are transmitted in a synchronization signal block (SS Block) occupying a concrete number of RBs per CBP. The PDCCH carries DCI signalling mapped in the CORESETs.

The reference signals in $5 \mathrm{G}$ NR has suffered changes compared to LTE. For instance, there is not Cell specific Reference Signal (C-RS) and a new Reference Signal PTRS has been introduced for Time/Frequency tracking. The signals used for channel estimation are the Demodulation Reference Signals (DMRS).

Another aspect to consider is channel coding. For user plane data, Low-Density Parity Check (LDPC) codes are adopted. Control channels are coded by means of a new channel coding technique based on the channel polarization concept [15]. Channel polarization brings a method for constructing capacity-achieving codes for binary input symmetric memoryless channels, as opposed to capacity approaching. It has been demonstrated that 5G-NR Polar codes outperform the Tail-Biting Convolutional Codes (TBCC) used in LTE control channels.

\section{A. Design of NR-MBMS for Terrestrial Broadcast}

The starting point taken in this document to development an NR-based Terrestrial Broadcast system was to begin by defining a number of new transmission modes in order to provide flexibility to cater for a wide range of deployment scenarios. With this starting point in mind, the following main design principles were then taken into account:

- Minimization of the footprint with respect to unicast transmission and scheduling processes. Reuse of unicast scheduling mechanism with one RNTI per service.

- Numerology options adequate for diverse scenarios including Multiple Frequency Network (MFN) and SFN configurations and topologies from low-power low-tower (LPLT) up to high-power high-tower (HPHT), with different inter-site distances.

- Transmission modes adequate for mobile (improving Doppler performance for high speed reception) and static reception (reducing capacity overheads at the expense of Doppler tolerance).

- Leveraging of 5G-NR bandwidth configuration and spectrum utilization efficiency to transmit large bandwidth signals instead of bandwidth-limited (to a few $\mathrm{MHz}$ ) carriers, if desired.

- Efficient multiplexing in time and frequency domain of local, regional and nation-wide services targeting mobile and fixed reception.

\section{1) Waveform and Numerology options}

The diverse nature of networks that may be used for transmitting Terrestrial Broadcast services (with network topologies ranging from LPLT to HPHT and from single-cell, MFN to nationwide SFN coverage areas) make it highly desirable to provide a range of new numerologies to better cater for the different types of transmission networks that could be used. Table II provides a set of numerologies that may be considered for Terrestrial Broadcast operation with different SCS, overhead and CP duration.

The reference numerology with $\mu=0$ (A) is already suitable for single-cell or MFN Terrestrial Broadcast operation, particularly from LPLT networks.

An extensive set of numerologies can be derived for SFN operation. Reference [13] introduced the concept of NRMBMS with extended CP by using a negative $\mu$ factor. This leads to SCS of $7.5 \mathrm{kHz}(\mu=-1), 3.75 \mathrm{kHz}(\mu=-2)$, etc. Examples derived with this method are B, C, D or F, highlighted in bold. However, although this mechanism may be useful to derive appropriate extended numerologies for LPLT deployments (note the similar numbers as for LTE), it is impractical to derive numerologies for SFN deployments with large-ISD (e.g. HPHT). These would require of longer OFDM symbol durations that may complicate implementation due to leading to non-integer multiples of current NR subframes. Moreover, following the same principle as in LTE, i.e. targeting mobile reception, the options are again limited to a few modes with $20 \%$ CP overhead.

Other numerologies may be derived following the method in [16] by re-defining the number of SCs per RB. Although initially proposed for LTE, the same mechanism can be applied to $5 \mathrm{G}-\mathrm{NR}$ as $15 \mathrm{kHz}$ SCS is also the standard numerology as well as $25 \mathrm{RBs}$ per $5 \mathrm{MHz}$ carrier $(4.5 \mathrm{MHz}$ effective bandwidth). Equation 1 provides the means to derive the new number of SC per RB as a function of the OFDM symbol duration and a $\mathrm{CP}$ fraction, which needs to be selected so that the number of SC per RB is an integer.

$$
S C_{R B}=12 \cdot 15 \mathrm{kHz} \cdot T_{S} \cdot(1-C P)
$$

By this method it is possible to derive multiple combinations of $T_{C P}$ and $T_{U}$. Note that OFDM symbol durations of $0.5,1,2,2.5,5$ and $10 \mathrm{~ms}$ are particularly interesting as an integer number of them fit into a $10 \mathrm{~ms}$ frame. Therefore, it is possible to select the appropriate combinations according to the deployment scenario and the receiving environment.

For instance, it is possible to select numerologies with acceptable mobility performance (i.e. wide SCS) and low capacity overhead for single-cell or MFN configuration (no 


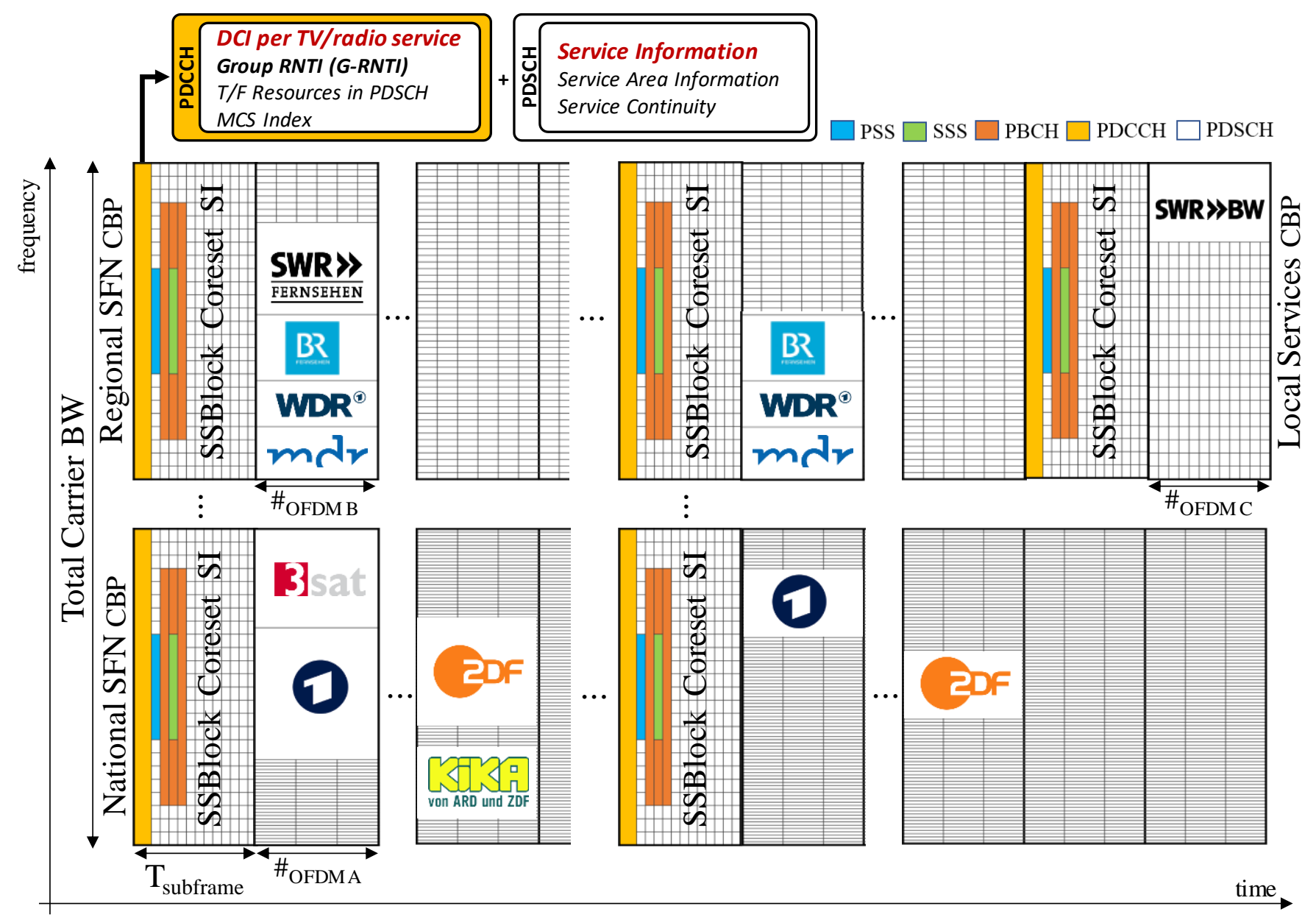

Fig. 2. The figure presents how the concepts of multiplexing numerologies within a given NR carrier to allocate services addressing different coverage requirements. Carrier Bandwidth Parts (FDM) or frames with different numerologies (TDM) could be used for the provision of Terrestrial Broadcast services. In the figure each service is transmitted in a different resource region according to the desired numerology. Note that for single-cell or MFN transmissions there is only one numerology $(15 \mathrm{kHz})$ employed. It is assumed that each service can be assigned a distinct DCI (G-RNTI, resource mapping and MCS index) therefore treating each TV/radio service in a similar way as user data in regular unicast frames. Note that over time services can be re-scheduled, activated and deactivated, according to broadcaster's demand.

need for a very large $\mathrm{CP}$ to cope with SFN echoes), numerologies with a large $\mathrm{CP}$ for moderate speed (e.g. those with $20 \%$ CP overhead), or numerologies with a large CP for SFN and static reception (with e.g. 4\% CP overhead). Numerology E could be useful for LPLT SFNs with a compromise between SFN gain in short/medium ISD deployments, while retaining high mobility. Numerology G corresponds to the one specified in FeMBMS.

It should be noted that although decreasing the $T_{C P} / T_{U}$ ratio reduces CP overhead, increasing the FFT size may cause significant impact in receiver complexity, which would need to be considered, along with other practical aspects of the receiver design.

Regarding reference signals, DMRS defined for unicast may be used for single-cell or MFN deployments, therefore with no changes. For SFN the most demanding channel may require the definition of new $\mathrm{RS}$, in particular denser in the frequency domain. Also for SFN, a common DMRS scrambling sequence for the stations involved in an SFN area is required.

\section{2) Bandwidth, Multiplexing and Spectrum Utilization}

For Terrestrial Broadcast deployments in frequencies below $1 \mathrm{GHz}, \mathrm{NR}$ frequency range FR1 provides the following options regarding bandwidth (frequency range FR1): 5, 10, 15, $20,25,40,50,60,80$ or $100 \mathrm{MHz}$. The bandwidth utilization in LTE was set to 90 percent in order to limit out-of-band emission produced by the slow decay of OFDM spectrum. This means that only $100 \mathrm{PRBs}(18 \mathrm{MHz})$ can be transmitted per 20 $\mathrm{MHz}$ bandwidth carrier. In NR, for the same case 106 PRBs (19.08 MHz) can be configured with $95.4 \%$ bandwidth utilization. Note that with numerology $15 \mathrm{kHz}$ a $50 \mathrm{MHz}$ carrier can be configured with 270 RBs $(97.2 \%$ bandwidth utilization). For $10 \mathrm{MHz}$ the bandwidth utilization is $93.6 \%$ (52 $\mathrm{RBs}$ ).

Note that the total allocated bandwidth can be extended via Carrier Aggregation, which in NR supports the bundling of up to 16 carriers. The combination of large bandwidth transmission with robust modulation and coding could also enable the introduction of Wideband Broadcasting transmission as presented in [17].

The potential application of the CBP concept to Terrestrial Broadcast distribution is proposed next. In this case, a single wideband carrier can multiplex not only services addressing different reception conditions, but also different coverage areas and network deployments. Fig. 2 shows a wideband carrier that allocates three different CBPs, each one with different numerology (see OFDM A, B and C). For instance, part of the 
BW can be reserved to schedule services for nationwide SFN coverage (OFDM A) alongside services intended for regional (OFDM B) or local (OFDM C) areas. A receiver would only demodulate the specific resources containing a desired service. Following this approach, a single 5G-NR carrier can transmit services intended for different reception conditions and coverage targets without the need of transmitting isolated multiplexes as currently done in Terrestrial Broadcast systems.

The use of CBPs is considered as an option for the FDM multiplexing of single-cell or MFN services with those intended for SFN areas within the same carrier. Note that different numerologies can also be multiplexed in TDM.

\section{3) Control Channel, Synchronization, Acquisition and Scheduling mechanism}

Signalling and synchronization mechanisms may be simplified with respect to FeMBMS, i.e. by reducing the amount of signalling to only that necessary for the correct reception and discovery of services. As system preambles, control channels are assumed to convey SS/PBCH Block, MIB signalling via the PDCCH and a series of SIBs via the PDSCH. Additional service announcement information may be allocated over the PDSCH so that physical channels and procedures do not require major modifications.

The characteristics of the SS/PBCH block and CORESET in NR not only provide higher flexibility but may also reduce the associated overhead.

On the one hand, a SS/PBCH block consists of 4 OFDM symbols in the time domain and 240 carriers in the frequency domain. A set of SS/PBCH block (namely SS burst) is transmitted according to the numerology, the frequency band and a periodicity of $5,10,20,40,80$, or $160 \mathrm{~ms}$ [18]. Hence, the largest capacity overhead for $\mathrm{SCS}=15 \mathrm{kHz}$, and frequency band below $6 \mathrm{GHz}$ is $2 \mathrm{SS} / \mathrm{PBCH}$ Blocks every frame (assuming a periodicity of $5 \mathrm{~ms}$ ), making a total of $2 \times 4 \times 240=1920$ REs per frame. These could be multiplexed in TDM/FDM with the necessary periodicity according to the nature of the services and the reception conditions (e.g. a high repetition rate is useful to minimize acquisition time when reception faces challenging conditions).

On the other hand, PDCCH flexibility of NR is enhanced with the introduction of CORESETs, which can be considered as smaller control units of former LTE Control Region. While LTE PDCCH is extended through the whole carrier bandwidth of OFDM symbols 1-3 of every subframe, NR PDDCH uses a reduced part of the carrier bandwidth used. One CORESET occupies from 1 to 16 Control Channel Elements (CCEs), comprising 6 Resource Element Groups (REGs), which in turn are composed by 12 REs. Hence, CORESET sizes go from $1 \times 6 \times 12=72$ REs to $16 \times 6 \times 12=1152$ REs per frame. 4 NRMBMS services with independent CORESETs, in a $50 \mathrm{MHz}$ bandwidth, would lead to a $2.78 \%$ overhead $^{3}$. Since longer periodicity values, e.g. $40 \mathrm{~ms}$, as well as larger carrier bandwidths are contemplated in NR, the associated overhead can be reduced.

\footnotetext{
$50 \mathrm{MHz}=270 \mathrm{RB} * 12 \mathrm{RE} / \mathrm{RB} * 14 \mathrm{OFDM}$ symb/subframe*10 subframes/frame $=453600 \mathrm{REs}$ in a frame.

$4 *(1152$ REs/CORESET + 1920 REs/SS-BCH burst $) * 100 / 453600=$ $2.78 \%$
}

Therefore, the existing mechanism in NR would have similar overhead to that in FeMBMS.

These control channels are proposed to be transmitted using regular unicast numerology as they may contain information which is intended to be transmitter-specific. By means of network planning the control channel resources for each transmitter can be scheduled so that co-channel interferences are avoided.

For single-cell or MFN transmissions as well as for SFN, coordinated frame scheduling is required in order to avoid overlaps between adjacent time/frequency resources that may create interference (in the first case) and to provide synchronous SFN transmission (in the latter case).

Although the mismatch between numerologies of the control channels and SFN data may still exist, it may be possible to detect the signal at the expense of larger detection time by means of a large aggregation level of e.g. PDCCH.

Regarding data scheduling, a reuse of the procedures existing for unicast are desired in order to minimize implementation impact and to exploit flexible resource allocation. A mechanism like the one used by SC-PTM in LTE could therefore be used. A G-RNTI could identify the resources allocated to broadcast in a similar way as userspecific content is identified with a C-RNTI. Furthermore, treating each $\mathrm{TV} /$ radio service in a similar way to unicast traffic, may make it possible to define a series of parameters for each specific TV/radio service. The transmitted service information would enable identification of time/frequency resource allocation in the frame, the corresponding MCS (with a corresponding DCI format), as well as service area information or service continuity information (by means of SIBs).

With dynamic scheduling, TV/radio services can be transmitted according to operator demands, exploiting better statistical multiplexing and spectral efficiency. Services could also be switched on and off or created (e.g. introduction of local services) over time, therefore only consuming the resources of the $5 \mathrm{G} \mathrm{NR}$ carrier when required.

Fig. 2 shows an illustrative example of a potential framing configuration. Note that some of the contents addressing different coverage areas (nationwide SFN, regional SFNs and local areas) are multiplexed within the same carrier.

\section{PERFormance EVAluAtion}

This section presents performance evaluation results of the proposed NR-MBMS system in comparison to LTE FeMBMS, focusing on the modes relevant for Terrestrial Broadcast and considering both single-cell or MFN modes and SFN modes. Special attention is given to the latter as these would require a different performance than for unicast modes.

The analysis is focused on spectral efficiency, robustness against frequency selectivity (e.g caused by natural multipath in single-cell or MFN configurations and artificial multipath due to echoes in an SFN) and time selectivity (due to Doppler shift for moving users). The analysis focusses on the channels conveying payload data (user plane). The link-level performance of the physical layer signaling (control plane) is also evaluated for different reception environments. Note that the results are obtained assuming realistic channel estimation 
TABLE III. SNR (DB) ACHIEVED IN THE CONSIDERED SCENARIOS.

\begin{tabular}{ccccc}
\hline \hline & TeChNOLOGY & $\begin{array}{c}\text { Mobile } \\
\text { (MCS2) }\end{array}$ & $\begin{array}{c}\text { Portable } \\
\text { (MCS12) }\end{array}$ & $\begin{array}{c}\text { Fixed } \\
\text { (MCS24) }\end{array}$ \\
\hline \multirow{3}{\text{SFN}}{$\begin{array}{c}\text { Modes } \\
\end{array}$} & FeMBMS & 7.0 & 11.4 & 20.5 \\
& NR-MBMS & 6.6 & 9.8 & 19.9 \\
\hline
\end{tabular}

with Least Square (LS) equalization and interpolating linearly in time domain and with an FFT-based method in frequency domain [20]. The selected Quality of Service (QoS) is a block error rate, BLER $<0.1 \%$.

For reference with LTE FeMBMS, performance is evaluated assuming that all resources in a frame are allocated to a given service (i.e. the maximum transport block size is selected according to the maximum number of resource blocks in a $5 \mathrm{MHz}$ bandwidth carrier). The frequency band considered is UHF $(700 \mathrm{MHz})$ as a traditional band of Terrestrial Broadcast systems.

Link-level simulations are complemented by coverage performance studies, which are mainly focused on new numerologies for SFN.

\section{A. Link-level Performance under Rician and Rayleigh fading}

Link-level performance is influenced by the channel and reception conditions that may hamper the correct reception of the signal. An adequate selection of the modulation and coding scheme (MCS) in both LTE FeMBMS and NR-MBMS systems implies a compromise between maximum spectral efficiency and SNR operation point.

This paper has considered three different types of reception conditions for Terrestrial Broadcast services according to [2], with their corresponding minimum SNR requirements. These are the following:

- Fixed roof-top reception, modelled by a Rician fading channel as per [19]. The minimum SNR requirement is considered to be $20 \mathrm{~dB}$.

- Portable reception, modelled by Rayleigh fading [19], with a minimum SNR requirement of $10 \mathrm{~dB}$.

- Mobile reception at a reference user speed of $60 \mathrm{~km} / \mathrm{h}$, modeled by a Typical Urban 6 (TU-6) channel model [19], with a minimum SNR requirement of $6 \mathrm{~dB}$.

Fixed reception benefits from Line-of-Sight (LoS) conditions, while portable reception introduces some loss due to the lack of it. Regarding mobile reception, different circumstances affect performance. Medium speed is generally the best case whereas low-speed reception lacks time selectivity and at high speed performance is degraded due to high Doppler shift.

Table III presents the SNR thresholds of the MCS indexes that are close to 6, 10 and $20 \mathrm{~dB}$ in FeMBMS and NR-MBMS for the three reception conditions. Note that, for comparison a SCS of $1.25 \mathrm{kHz}$ is considered for both systems.

As it can be observed, NR-MBMS provides a performance gain in all cases, although the improvement is below $1 \mathrm{~dB}$. This gain comes from an advanced physical layer with LDPC decoding.

Single-cell or MFN configuration, which can be implemented using the regular $15 \mathrm{KHz}$ SCS mode, may experience similar gains. In this case, SC-PTM can be

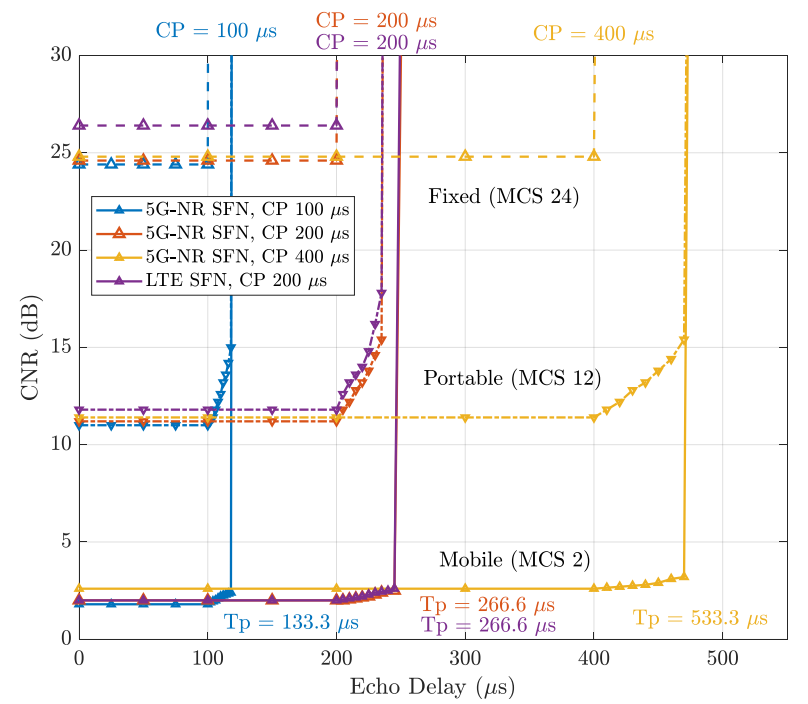

Fig. 3. Required CNR performance for different MCS and echo delays in Terrestrial Broadcast and LTE FeMBMS.

compared to the proposed NR-MBMS MFN modes. In terms of performance, there are differences in the order of $1 \mathrm{~dB}$ for robust MCS modes whereas the difference decreases for high MCS indexes. As an example, the required CNR threshold in AWGN conditions for SC-PTM is $-3.2 \mathrm{~dB}$ (MCS2), $5 \mathrm{~dB}$ (MCS12) and $15.5 \mathrm{~dB}$ (MCS24) whereas for NR-MBMS with a single-cell/MFN mode the values are $-4.1 \mathrm{~dB}, 5.1 \mathrm{~dB}$ and $15.7 \mathrm{~dB}$, respectively [22].

\section{B. Link-Level Performance with echoes in SFN networks}

NR-MBMS performance is evaluated in frequency selective fading environments, where SFN multipath may represent a degradation. In this case, the performance is represented as the variation of the minimum required $\mathrm{CNR}$ depending on the relative echo delay.

The SFN scenario is modelled by extending the $0 \mathrm{~dB}$ echo channel in [19]. This channel is defined as a channel model formed by two paths with same amplitude and a time delay between them equivalent to $90 \%$ of the CP duration. The second path is moved in order to set different delays inside and outside the $\mathrm{CP}$.

Fig. 3, focused on ISD from $30 \mathrm{~km}$ to $120 \mathrm{~km}$ (e.g. HPHT networks), shows the performance for different echo delays for the three MCS indexes defined in Table III. As it can be observed, the required CNR remains constant when artificial echoes arrive inside the $\mathrm{CP}$ region. When the echoes arrive out of the $\mathrm{CP}$ region, a performance degradation begins until the Nyquist limit $(T p)$, which limits the system operation. Comparing the different configurations, it can be observed that NR-MBMS outperforms LTE FeMBMS in terms of SFN performance. Special focus is given to the performance of the system for echoes arriving after $\mathrm{CP}$ and before $T p$ (i.e. within the equalization interval). Robust enough MCS indexes permit to extend system performance beyond CP. In such case, a proper design of the reference signals for SFN networks is critical as low CPs not stressing the framing design of the system could still be used in SFN deployments. These results are aligned with the conclusions presented in [21].

For SFN deployments with short ISD (e.g. LPLT networks), 

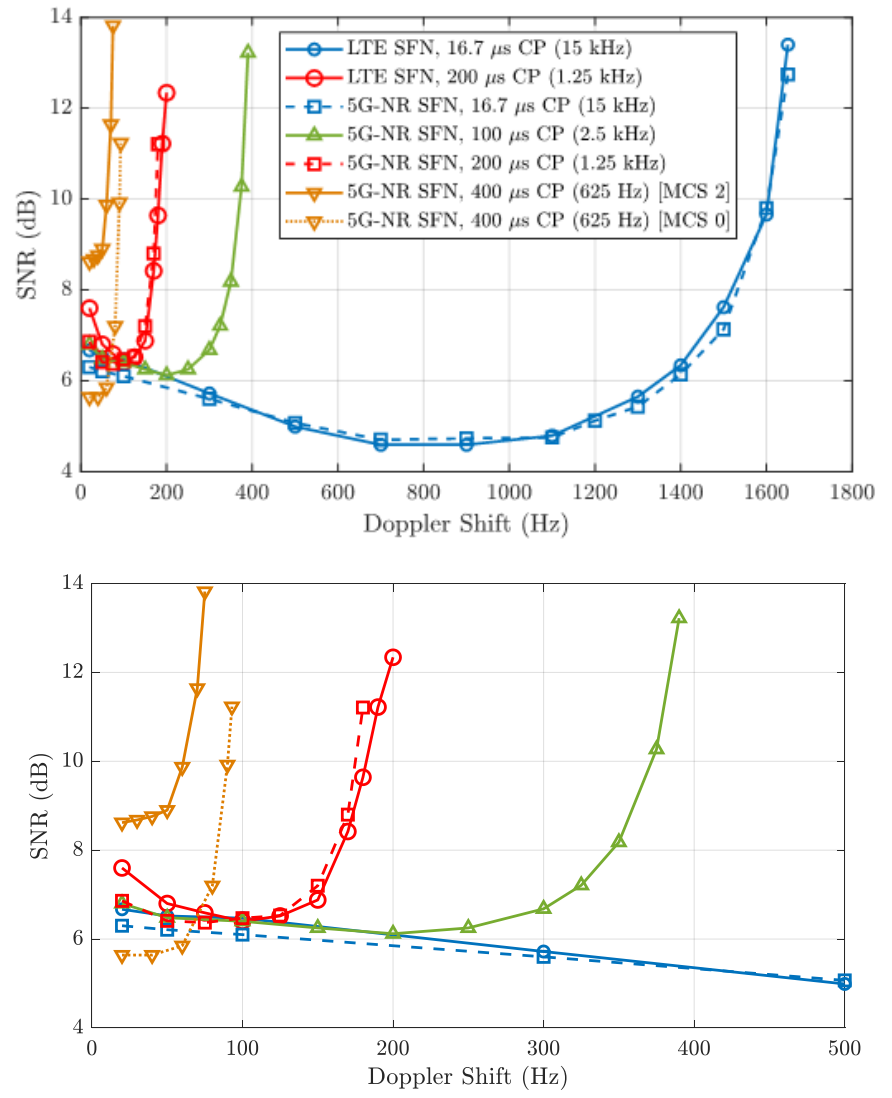

Fig. 4. Doppler shift (Hz) vs. SNR (dB) when using the different SCS options in both LTE FeMBMS and NR-MBMS. Complete range of Doppler Shift from 0 to $1800 \mathrm{~Hz}$ (top) and zoom from 0 to $500 \mathrm{~Hz}$ (bottom).

reference [22] provides link level performance results for numerologies with extended $\mathrm{CP}$ and negative factor $\mu$ ( $\mu=0$, $\mu=-1$ and $\mu=-2$ ). In general, robust MCS can provide better resilience against $\mathrm{SFN}$ echoes outside the $\mathrm{CP}$.

\section{Resilience to Doppler shift at high speed and SFN deployments}

This section studies the tolerance to Doppler spread with practical receiving algorithms in both FeMBMS and NRMBMS following the procedures in [22] and [23] as a reference.

Doppler shift is a natural effect of the relative speed between users and transmitters. OFDM systems are affected by this phenomenon which is directly related to SCS. Therefore, the effect is critical for mobile reception in SFN deployments, in particular when large ISD need to be covered. Single-cell or MFN modes may also suffer from this effect when low overhead configurations are used (i.e for low CP to $T_{U}$ ratios).

Channel estimation assisted by reference signals in OFDM systems relies on measurements made on those subcarriers which are reference-bearing signals. This can be performed every $D_{t}$-th symbol. Since symbols occur at the rate $f_{s}=1 /\left(T_{U}+T_{C P}\right)$, it follows that the Nyquist limit for temporal channel variation (i.e. Doppler limit) that can be measured is [19]:
TABLE IV. THEORETICAL DOPPLER LIMIT AND MAXIMUM USER SPEED AT $700 \mathrm{MHz}$.

\begin{tabular}{ccccc}
\hline \hline SCS & $625 \mathrm{~Hz}$ & $1.25 \mathrm{kHz}$ & $2.5 \mathrm{kHz}$ & $15 \mathrm{kHz}$ \\
\hline$T_{C P}(\mu \mathrm{s})$ & 400 & 200 & 100 & 16,67 \\
$T_{U}(\mu \mathrm{s})$ & 1600 & 800 & 400 & 66,67 \\
$D_{t}$ & 2 & 2 & 2 & 3 \\
Doppler limit (Hz) & 125 & 250 & 500 & 2000 \\
Max. user speed @ & 192 & 384 & 771 & 3085 \\
\hline $700 \mathrm{MHz}(\mathrm{km} / \mathrm{h})$ & \multicolumn{2}{c}{1} & & \\
\hline$f_{D_{\text {limit }}}=\frac{2}{2 D_{t}\left(T_{U}+T_{C P}\right)} \mathrm{Hz}$ & &
\end{tabular}

Hence, the performance depends on the subcarrier spacing, system bandwidth, the operational frequency band and the accuracy of channel estimation method used.

A wide range of Doppler shifts is evaluated, which can later be mapped to a given receiver speed according to the assumed frequency. Note that for single-cell or MFN configurations using a wide subcarrier spacing (e.g. $15 \mathrm{kHz}$ ) Doppler may not impose a critical limit. Therefore, theoretical Doppler limits for different SFN configurations are shown in Table IV. Reference signals with $D_{t}$ values $3(15 \mathrm{kHz})$ and 2 (extending $1.25 \mathrm{kHz}$ to $2.5 \mathrm{kHz}$ and $625 \mathrm{kHz}$ ) are assumed.

These theoretical values are evaluated considering realistic scenarios modelled by a TU-6 channel with variable speed. The Doppler shift limit is calculated as the value that entails a CNR performance loss of $3 \mathrm{~dB}$ compared to the lowest CNR achieved in the whole range [24].

Fig. 4 shows how the use of a different numerology has a great impact on the mobility tolerance. However, in general the results practically do not change between LTE FeMBMS and NR-MBMS if this parameter is the same. For instance, both LTE and NR with SCS $15 \mathrm{kHz}$ allow Doppler shifts up to $1550 \mathrm{~Hz}$, equivalent to $2390 \mathrm{~km} / \mathrm{h}$ at $700 \mathrm{MHz}$. The common SCS that can be selected in both LTE FeMBMS and NRMBMS, i.e. $1.25 \mathrm{kHz}$, reduces the maximum Doppler shift to $180 \mathrm{~Hz}$ approximately, equivalent to $280 \mathrm{~km} / \mathrm{h}$ when MCS 2 is selected. A higher MCS may provide lower correction capabilities, thus lowering this value. It should also be noticed that compared to FeMBMS, the user speeds permitted with SCS $625 \mathrm{~Hz}$ (CP $400 \mu \mathrm{s}$ ) are considerably lower. This mode has been designed to support high-demanding coverage requirements in fixed reception scenarios, which implies the use of long CPs that, in turn and in order to minimize overheads, require narrow SCS. This mode permits $75 \mathrm{~Hz}$ Doppler shift, which represents a user speed of $115 \mathrm{~km} / \mathrm{h}$ with MCS 2.

The use of a more robust MCS (e.g. MCS index 0) reduces the SNR at low user speeds. However, the maximum Doppler shift permitted is still $75 \mathrm{~Hz}$. Overall, improved performance is achieved at the expense of capacity when using robust transmission modes.

One important drawback of LTE FeMBMS and NR-MBMS is the lack of time interleaving which will improve performance by increasing time diveristy. With time 


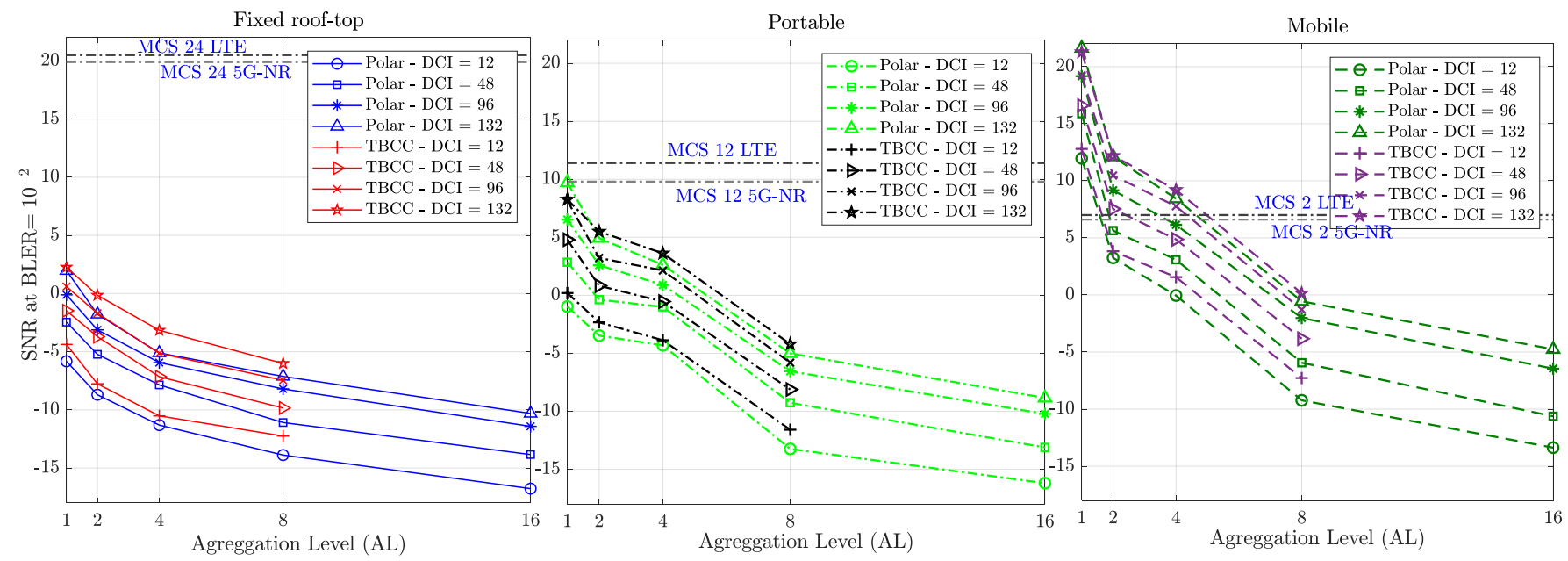

Fig. 5. 5G-NR polar codes and LTE TBCC codes performance for different DCI lengths $(12,48,96,132)$ and Aggregation Levels (1, 2, 4, 8, 16 for Polar and 1, 2, 4, 8 for TBCC) in Fixed (left), Portable (center) and Mobile (right) reception conditions.

interleaving, the system could withstand user speeds higher than $250 \mathrm{~km} / \mathrm{h}$, as shown in [25]. The SCS of $2.5 \mathrm{kHz}$ represents a compromise between both options. In this case, SFN coverage may suffer with large ISD, but mobility is still relatively high, i.e. $390 \mathrm{~Hz}$ equivalent to $600 \mathrm{~km} / \mathrm{h}$ at 700 $\mathrm{MHz}$.

\section{Link-level performance of Control Channels}

This section analyses the performance of the coding schemes employed in LTE and 5G-NR PDCCH, as this is detected as one of the most limiting channels from those constituting the FeMBMS CAS [11]. 3GPP has adopted Polar coding for control channel in $5 \mathrm{G}-\mathrm{NR}$, which reduce decoding complexity while almost closing the gap to Shannon. LTE employs TBCC (Tail Biting Convolutional Code) instead.

In order to increase the decoding probability of the $\mathrm{PDCCH}$ (i.e. decoding of DCI formats), LTE and NR define different Aggregation Levels (AL). According to the selected DCI format, it is possible to define an AL so that the scheduler will define an appropriate robustness increase. There is a trade-off between robustness and data rate. The higher the AL, the higher the number of subcarriers used to transmit a DCI which, at the same time, limits the number of DCIs that can be allocated into a given subframe. While LTE permits to use an $\mathrm{AL}$ up to $8,5 \mathrm{G}-\mathrm{NR}$ has increased this value up to 16 repetitions. This was adopted for allowing the correct demodulation even at very high noisy conditions.

Fig. 5 presents the performance of both $\mathrm{PDCCH}$ coding schemes for a wide range of DCI lengths and AL over fixed, portable and mobile reception conditions. This validates that 5G-NR Polar codes outperform LTE TBCC. In particular, the performance gains for $\mathrm{DCI}=12$ bits and $\mathrm{AL}=8$ are between $1.6 \mathrm{~dB}$ and $2 \mathrm{~dB}$ for the different reception scenarios under evaluation. It can be observed that doubling the AL reduces approximately in $3 \mathrm{~dB}$ the SNR requirements. The shortest DCI length (i.e. DCI = 12) is the most robust one, thanks to having the lower effective coding rate. The possibility to increase AL up to 16 brings an advantage which may eliminate the potential problems for detecting the CAS in LTE.
TABLE V. COVERAge SimUlation PARAMETERS

\begin{tabular}{cc}
\hline \hline Parameter & Roof-Top Reception \\
\hline Receiving Antenna Height & $10 \mathrm{~m}$ \\
Receiver Noise Figure & $6 \mathrm{~dB}$ \\
Rx Antenna Pattern & ITU-R BT.419 \\
Rx Antenna Gain & $13.15 \mathrm{dBi}$ \\
Antenna Cable Loss & $4 \mathrm{~dB}$ \\
Implementation Margin & $1 \mathrm{~dB}$ \\
Noise Bandwidth & $4.5 \mathrm{MHz}$ \\
Frequency & $700 \mathrm{MHz}$ \\
Propagation Model & ITU-R P.1546-5 over land \\
Wanted Signal Time Value & $50 \%$ time \\
Interfering Signal Time Value & $1 \%$ time \\
Location Variation & $5.5 \mathrm{~dB}$ (log-normal distribution) \\
Signal Summation & Schwartz \& Yeh power sum \\
Pixel size & $100 \mathrm{~m}$ x 100m
\end{tabular}

\section{E. Coverage evaluation for SFN networks}

An overview on the suitability of enhancing numerologies for SFN networks is provided in this section. Wide area SFNs have been modelled using an hexagonal network layout with five rings of sites around a central transmitter. The available SINR, incorporating the effects of SFN self-interference, has been computed at an apex of the central hexagon.

In the LPLT networks the effective radiated power (ERP) was set to $40 \mathrm{~W}$ at an effective antenna height of $30 \mathrm{~m}$ while 50 $\mathrm{kW}$ and $250 \mathrm{~m}$ were used for the HPHT network.

Table $\mathrm{V}$ sets out the receiving environment parameters used in the simulations; all values are in-line with [26]. ITU-R P.1546-5 has been used to calculate the mean signal strengths of the wanted and interfering signals in $100 \mathrm{~m} \times 100 \mathrm{~m}$ 'pixels' comprising the coverage area. Within a pixel these signals vary from one location to another according to a log-normal distribution with standard deviation of $5.5 \mathrm{~dB}$ and has thus been modelled as random variables. The Schwartz and Yeh method has been used to calculate the combined wanted and interfering signal powers so that the probability of reception at any point within the pixel can be determined.

A generic analysis of the coverage for fixed roof-top reception has been conducted as a function of ISD for various different CP lengths $(33,100,200,300$ and $400 \mu$ s) where the 
TABLE VI. Percentage of UK Households at Percentage Locations

\begin{tabular}{cccccc}
\hline \hline $\begin{array}{c}\Delta_{f} \\
(\mathrm{~Hz})\end{array}$ & $T_{U}(\mu \mathrm{s})$ & $\begin{array}{c}T_{C P} \\
(\mu \mathrm{s})\end{array}$ & $\begin{array}{c}T_{S} \\
(\mathrm{~ms})\end{array}$ & $\begin{array}{c}\text { ISD } \\
(\mathrm{km})\end{array}$ & $\begin{array}{c}\text { Coverage } \\
\%\end{array}$ \\
\hline 1250 & 800.00 & 200.00 & 1.0 & 60 & 86.5 \\
625 & 1600.00 & 400.00 & 2.0 & 120 & 98.1 \\
208.33 & 4800 & 200 & 5.0 & 60 & 97.6 \\
217.39 & 4600 & 400 & 5.0 & 120 & 99.3 \\
\hline \hline
\end{tabular}

two latter CPs have been hypothecated in order to determine whether there would be any benefit in further extending the CP. For these two modes the OFDM symbol period has been extended accordingly so that the $\mathrm{CP}$ always represents $1 / 4$ of the symbol duration - in line with the standardized eMBMS modes and those initially proposed for NR-MBMS. The achievable SINR, at the apex of the central hexagon in the network was then computed for reception qualities of $70 \%$ and $95 \%$ locations, as two common metrics for coverage estimation.

Coverage quality is then expressed as the percentage of locations exceeding a given SINR threshold within a pixel for $99 \%$ of the time.

Fig. 6 presents the results for LPLT (top) and HPHT (bottom) networks. It is found that for all the LPLT ISDs studied, the $200 \mu$ s CP would be sufficiently long. Extending it further would provide no additional benefit against SFN selfinterference - the achievable SINR would not increase. Conversely it can be seen that the $200 \mu$ s CP significantly improves the SINR for all the LPLT ISDs studied compared with the $33 \mu$ s option while a $100 \mu$ s variant may be a good addition for networks with ISDs of 5 to $10 \mathrm{~km}$.

For HPHT networks, it can be seen that the $200 \mu \mathrm{s}$ CP for ISDs greater than $70 \mathrm{~km}$ - i.e. ISDs typical of existing Terrestrial Broadcast networks - is too short. The introduction of longer CPs would improve the coverage of the system. Here a $300 \mu$ s and $400 \mu$ s CPs have been computed, being the latter the one providing the highest improvement.

According to the results, wide area coverage in existing Terrestrial Broadcast networks - where ISDs of $60 \mathrm{~km}$ or more are common - may be limited to modes with SINR thresholds below 12-13 dB for $95 \%$ coverage availability, or below $19 \mathrm{~dB}$ for $70 \%$ coverage availability.

The coverage of eMBMS in a national SFN is now assessed in the UK Terrestrial Broadcast network in order see what may happen in a more practical setting.

In this example the UK Prediction Model (UKPM) was used - a prediction model jointly developed by ITC, BBC, Crown Castle and NTL for planning Terrestrial Broadcast services in the UK [27]. All 1100+ UK Terrestrial Broadcast transmitters were modelled with the eMBMS parameters shown in Table V. All other physical characteristics of the network, such as antenna patterns, ERPs, transmitter locations and antenna heights were otherwise unchanged.

It is clear from Table VI that the $200 \mu \mathrm{s} C \mathrm{C}$ would be too short to achieve near-universal coverage with a national SFN. Although this result is somewhat different to the hexagonal network simulations, it may be explained by observing that practical networks are much less regular. For example, they contain real terrain and ISDs of various lengths, some greater than $60 \mathrm{~km}$. Sea paths over convex sections of coast also lead to higher interference than is found in the land based regular
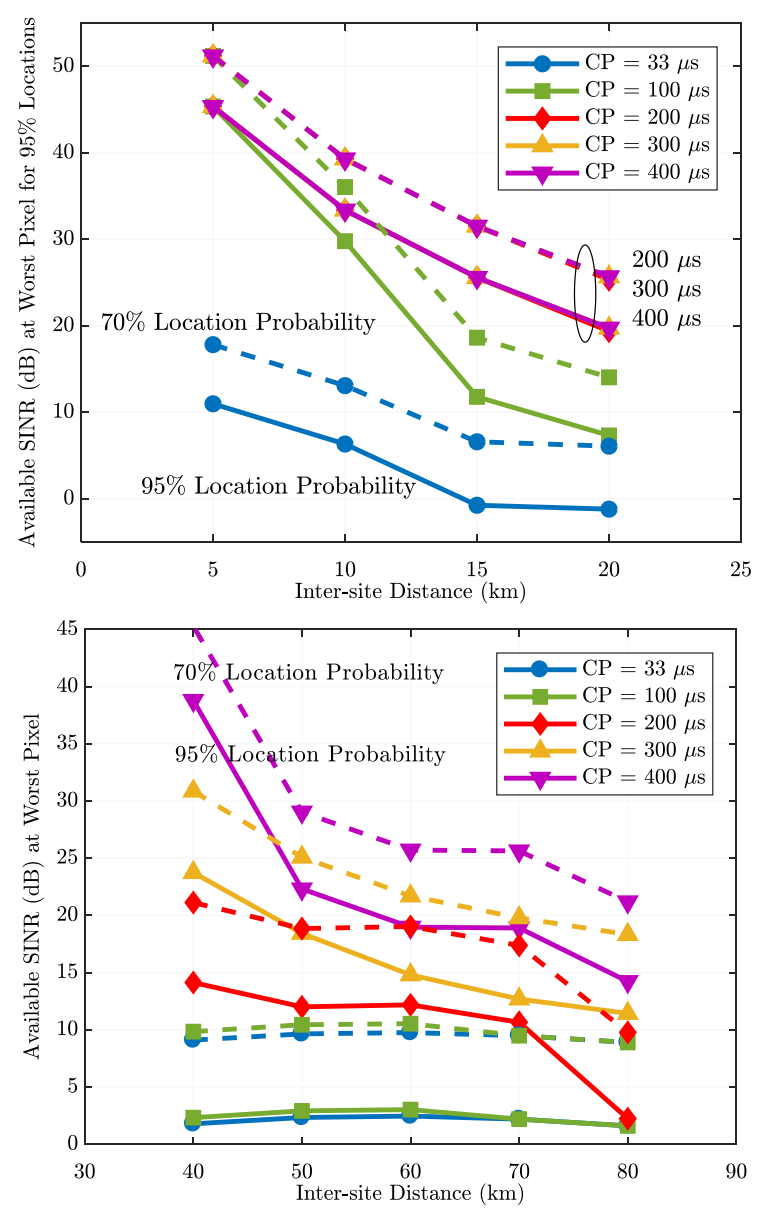

Fig. 6. Available SINR at the worst pixel of the LPLT and HPHT networks as a function of the ISD and different CP duration (SFN).

hexagon networks. A longer $\mathrm{CP}$, in the order of $400 \mu$ s, would therefore be beneficial.

Simulation for additional modes with 200 and $400 \mu$ s CP but with significantly larger OFDM symbol durations have also been carried out. A clear benefit is achieved in both cases where $11.1 \%$ and $1.2 \%$ more coverage is reached in comparison with the respective modes with $20 \%$ overhead.

Overall, the network may benefit from an SINR increase together with larger capacity thanks to lower overheads.

\section{CONCLUSIONS}

The design of the air interface of an MBMS system based on 5G New Radio (NR), NR-MBMS, has been outlined. The design extends the recent 5G-NR Release 15 and Release 16, initially only focused on unicast transmissions, to Terrestrial Broadcast services. The design does not necessarily require a split between a mixed mode carrier containing unicast/ multicast/broadcast or a dedicated carrier as the latter is simply derived from the allocation of $100 \%$ of resources to Terrestrial Broadcast services.

For the single-cell or MFN configurations, the physical layer design that has been outline has a minimal impact with respect to unicast. Existing synchronization and acquisition mechanisms are could be reused with only minor changes. Linear TV/radio services can be allocated by means of a group identifier (G-RNTI) in a similar fashion as unicast data is 
scheduled. LPLT (small cells) as well as HPHT (large cells) stations can be employed. The NR carrier may be used to allocate up to $100 \%$ broadcast data multiplexed in both time and frequency domains with high granularity and without major constraints (by reusing the existing procedures for unicast).

SFN may be enabled by extending the single-cell mode which may require a more complex design in terms of numerologies and a corresponding trade-off between mobility and SFN coverage. Note also that MFN numerologies may also be optimized to reduce capacity overheads. It is also important to note that although it is desirable from a deployment perspective to have as much flexibility as possible, consideration should also be given on the potential receiver complexity and testing that may impose limitations on the maximum number of numerology options finally included in the specifications.

Based on 5G NR, the system outlined herein may outperform the existing FeMBMS system (based on LTE). The design takes into account different reception scenarios targeting high speed (at expense of capacity overhead) and static reception (maximizing SFN efficiency and capacity). The use of the new physical layer features of 5G-NR such as new LDPC and Polar codes, increased bandwidth efficiency or efficient numerology multiplexing permits the configuration of new transmission mechanisms that outperform FeMBMS. 5G-NR may have up to $7.2 \%$ higher bandwidth utilization compared with FeMBMS. With the use of bandwidth parts, a single wideband carrier can multiplex services intended for different reception conditions and also different coverage areas, including local, regional SFN and nation-wide SFN. Data channels can benefit from approximately $0.5 \mathrm{~dB}$ gain in CNR threshold whereas the gain in term of control channels is more noticeable thanks to the possibility of increasing aggregation levels. The existing control channels for unicast may already enable reduced signaling overhead with respect to the CAS in FeMBMS and may not require any modification as they are more flexible in terms of resource allocation and periodicity. In terms of overheads, a skillful design may be possible to maximize capacity by an adequate $\mathrm{CP}$ and useful OFDM symbol duration.

Common techniques used in other standards, such as physical layer time interleaving for improved robustness in mobile environments, would also be of benefit, should they be adopted by $5 \mathrm{G}-\mathrm{NR}$ Terrestrial Broadcast.

\section{REFERENCES}

[1] 3GPP TR 38.913 v14.3.0, "Study on scenarios and requirements for next generation access technologies," June 2017.

[2] D. Vargas and D. Mi, Eds., "LTE-Advanced Pro Broadcast Radio Access Network Benchmark," Deliverable D3.1, 5G-PPP 5G-Xcast project, Nov. 2017.

[3] I. Eizmendi et al. "DVB-T2: The second generation of terrestrial digital video broadcasting system" IEEE Trans. Broadcast., vol. 60 no. 2 pp. 258-271, June 2014.

[4] L. Fay, L. Michael, D. Gomez-Barquero, N. Ammar, and M. W. Caldwell "An overview of the ATSC 3.0 physical layer specification", IEEE Trans. Broadcast., vol. 62 no. 1 pp. 159-171, March 2016.

[5] ETSI EN 300401 V1.4.1" "Radio Broadcasting Systems; Digital Audio Broadcasting (DAB) to mobile portable and fixed receivers", June 2006.

[6] HbbTV 2.0.2 Specification, HbbTV Association, December 2018.
[7] 3GPP TR 36.776 V1.1.0, "Study on LTE-based 5G Terrestrial Broadcast (Release 16)", March 2019.

[8] P. Klenner, J. Baek, N. S. Loghin, D. Gomez-Barquero, and W. Ko, "Physical Layer Time Interleaving for the ATSC 3.0 System," in IEEE Trans. Broadcast., vol. 62, no. 1, pp. 253-262, March 2016.

[9] L. Zhang et al., "Layered-Division-Multiplexing: Theory and Practice," in IEEE Trans. on Broadcast., vol. 62, no. 1, pp. 216-232, March 2016.

[10] 3GPP TR 36.859 V13.0.0, "Study on Downlink Multiuser Superposition Transmission (MUST) for LTE", January 2016.

[11] J. J. Gimenez, P. Renka, S. Elliott, D. Vargas and D. Gomez-Barquero, "Enhanced TV Delivery with eMBMS: Coverage Evaluation for RoofTop Reception," 2018 IEEE International Symposium on Broadband Multimedia Systems and Broadcasting (BMSB), Valencia, 2018, pp. 1-5.

[12] L. Richter and S. Ilsen, "Coverage Evaluation of LTE FeMBMS: a Case Study Based on a DVB-T2 Network," 2018 IEEE International Symposium on Broadband Multimedia Systems and Broadcasting (BMSB), Valencia, 2018, pp. 1-5.

[13] 3GPP R1-1712879: "Forward Compatibility Consideration for ECP Design and NR-MBMS", August 2017.

[14] EBU Tech. Rep. 3348, "Frequency and Network Planning Aspects of DVB-T2," Oct. 2014

[15] E. Arikan, "Channel Polarization: A Method for Constructing CapacityAchieving Codes for Symmetric Binary-Input Memoryless Channels," in IEEE Transactions on Information Theory, vol. 55, no. 7, pp. 30513073, July 2009.

[16] G. K. Walker, X. Zhang and X. F. Wang, "Extended duration cyclic prefix with low overhead for LTE Broadcast" U.S. Patent 2015/0078292 A1, issued March 19, 2015.

[17] J. J. Gimenez, D. Gomez-Barquero, J. Morgade and E. Stare, "Wideband Broadcasting: A Power-Efficient Approach to 5G Broadcasting," in IEEE Communications Magazine, vol. 56, no. 3, pp. 119-125, March 2018.

[18] 3GPP TR 38.213 V15.4.0, "NR; Physical Layer Procedures for control", January 2019

[19] ETSI TS 102831 v1.2.1, "Digital Video Broadcasting (DVB); Implementation guidelines for a second generation digital terrestrial television broadcasting system (DVB-T2)", August 2012

[20] L. Zhang, et al., "Enhanced DFT based channel estimation for LDM systems over SFN channels," in Proceedings IEEE International Symposium on Broadband Multimedia Systems and Broadcasting (BMSB), Ghent, Belgium, June 2015.

[21] J. L. Carcel, J. J. Gimenez and D. Gomez-Barquero, "Zero-guard OFDM performance in SFN with ATSC 3.0 ultra-robust transmission modes," 2017 IEEE International Symposium on Broadband Multimedia Systems and Broadcasting (BMSB), Cagliari, 2017, pp. 1-5.

[22] E. Garro, M. Fuentes, J. J. Gimenez, J. L. Carcel, Eds., "Air Interface", 5G-Xcast Deliverable D3.2, November 2018.

[23] M. Fuentes, et. al., "Physical Layer Performance Evaluation of LTEAdvanced Pro Broadcast and ATSC 3.0 Systems," to appear at IEEE Trans. Broadcasting.

[24] S. I. Park et al., "Low Complexity Layered Division Multiplexing for ATSC 3.0," IEEE Trans. on Broadcast., vol. 62, no. 1, pp. 233-243, March 2016.

[25] Zhang, et. al., "Using Layered-Division-Multiplexing to Deliver MultiLayer Mobile Services in ATSC 3.0," IEEE Trans. Broadcasting, vol. 65, no. 1, March 2019.

[26] EBU, "Simulation Parameters for Theoretical LTE eMBMS Network Studies", Tech. Rep 034.

[27] P. G. Brown, K. T. Tsioumparakis, M. Jordan, A. Chong, "UK Planning Model for Digital Terrestrial Television Coverage," BBC Research \& Development, White Paper WHP 048, Sept. 2002.

[28] D. Gomez-Barquero, D. Navratil, S. Appleby and M. Stagg, "Point-toMultipoint Communication Enablers for the Fifth-Generation of Wireless Systems", IEEE Communications Standards Magazine, vol. 2, no. 1, March 2018.

[29] A. Awada, M. Säily and L. Kuru, "Design and performance impact of long cyclic prefixes for eMBMS in LTE networks," 2016 IEEE Wireless Communications and Networking Conference, Doha, 2016, pp. 1-7. 